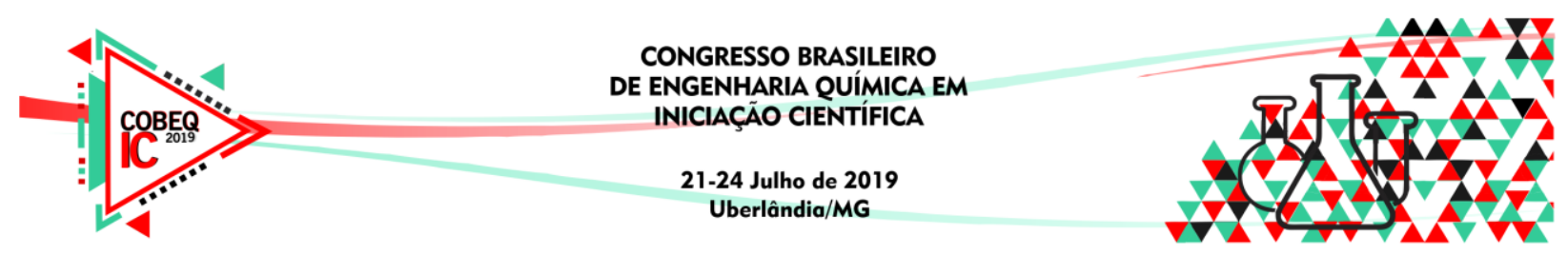

\title{
CONTRIBUIÇÃO AO ESTUDO DO TRATAMENTO DE RESÍDUOS PLÁSTICOS VIA DEGRADAÇÃO TÉRMICA COM ÁGUA SUPERCRÍTICA
}

\author{
F.S. PULCHERIO ${ }^{1}$, M.Y. MARTINS ${ }^{1}$, R.B.R. SILVA ${ }^{1}$, J.A. RIBEIRO ${ }^{2}$ \\ ${ }^{1}$ Universidade Federal de Mato Grosso, Faculdade de Engenharia Química, (discente) \\ ${ }^{2}$ Universidade Federal de Mato Grosso, Faculdade de Engenharia Química, (docente) \\ E-mail para contato: grupooxide@gmail.com
}

\begin{abstract}
RESUMO - Os polímeros são hidrocarbonetos de cadeias muito longas e elevado peso molecular, compostos de estruturas menores conhecidas como monômeros. $\mathrm{O}$ processo que forma os polímeros recebe o nome de polimerização. Todavia, existem inúmeros meios para a despolimerização dessas estruturas, esse trabalho tratará da degradação térmica com água supercrítica, fenômeno esse que se inicia com a elevação da temperatura e pressão da água aos níveis supercríticos, de modo que se torne um solvente de composto apolares, sendo ideal para a interação com os hidrocarbonetos. Os polímeros estudados para o tratamento serão plásticos, sendo assim a medida é também um método de reciclagem, o qual utiliza água como solvente, sendo limpo em termos de tecnologia. Portanto, o projeto soma não só com o conhecimento adquirido, mas também com práticas sustentáveis e Química Verde, visto que é possível a obtenção de um conjunto de moléculas orgânicas pequenas partindo de resíduos plásticos.
\end{abstract}

\section{INTRODUÇÃO}

Os polímeros sintéticos, conhecidos como plásticos estão incorporados ao cotidiano das pessoas de diversas formas: embalagens, brinquedos, móveis, computadores, calçados e automóveis são alguns exemplos. Além de práticos, são impermeáveis, maleáveis, duráveis e com uma excelente relação custo/benefício e contribuem para o desenvolvimento econômico. Porém, a maioria dos plásticos comumente usados são derivados do petróleo e não são biodegradáveis, assim, quando consumidos em grande escala podem gerar sérios problemas ambientais.

Segundo a revista americana "Science Advances", de 1950 até 2015, os seres humanos geraram 6,3 bilhões de toneladas (t) de resíduos plásticos, sendo que, apenas $9 \%$ desses resíduos foram reciclados. Além disso, ainda segundo a revista, até 2050, 9.000 t de resíduos plásticos terão sido recicladas, $12.000 \mathrm{t}$ incinerados e $12.000 \mathrm{t}$ descartados em aterros sanitários ou no ambiente natural.

Existem vários métodos para a reutilização e reciclagem de plásticos que ajudam a dar outros destinos para o que, atualmente, é considerado lixo. O objeto de investigação deste trabalho é o processo de degradação térmica utilizando água supercrítica como solvente. Este 


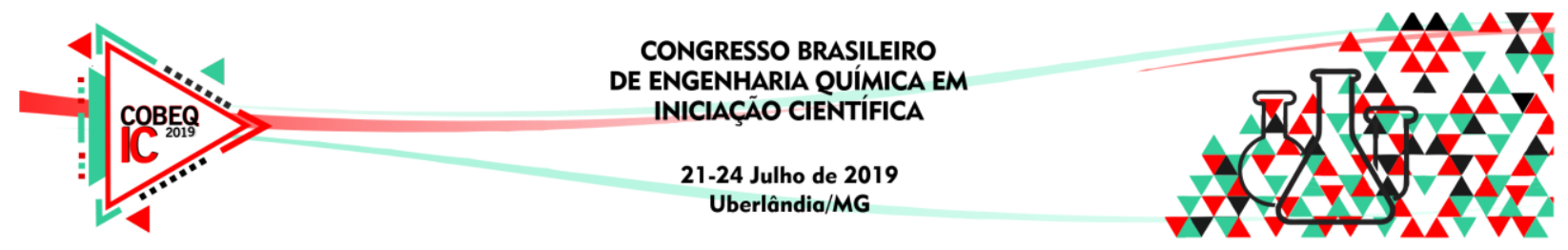

tratamento quebra a molécula polimérica em um conjunto de moléculas menores que possuem valor agregado, possibilitando assim, sua reutilização ou até mesmo sua total degradação.

\section{OBJETIVOS}

O objetivo deste trabalho é o estudo do processo de degradação térmica de resíduos plásticos a partir do processo oxidativo com o uso de água supercrítica, como tentativa de solucionar um problema urbano atual. $\mathrm{O}$ escopo deste trabalho se baseia na revisão da metodologia atualmente utilizada.

\section{METODOLOGIA}

Um fluido supercrítico (FSC) é definido como um fluido onde a temperatura e pressão estão acima de seus pontos críticos termodinâmicos. O ponto crítico representa um estado único, estabelecido pelas interações moleculares específicas de cada substância e é matematicamente descrito como o ponto onde a primeira e segunda derivada da isoterma Tc é igual a zero, conforme ilustrado na Figura 1. Para um composto puro, o ponto crítico ocorre no limite do equilíbrio vapor-líquido e acima deste ponto a substância não é considerada unicamente líquido ou gás, e sim um homogêneo fluido supercrítico. As condições particulares (pressão e temperatura) de uma substância que atingiu seu estado supercrítico são referidos como constantes críticas (KORETSKY, 2007).

Figura 1 - Comportamento PV de equações de estado cúbicas

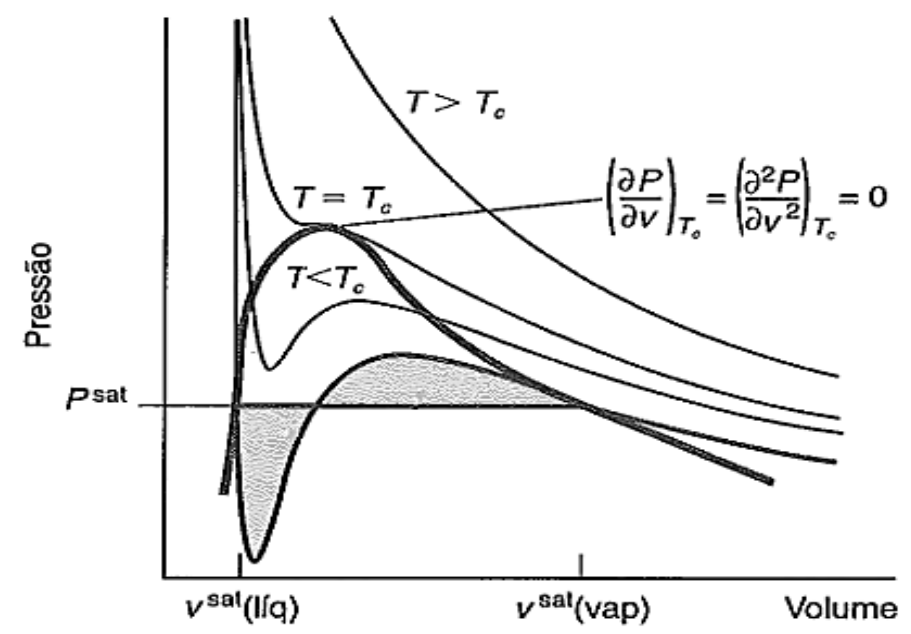

Fonte: Termodinâmica para Engenharia Química.

Comparada a outras substâncias a água possui temperatura e pressão críticas relativamente altas sendo elas respectivamente $374^{\circ} \mathrm{C}$ e 221,2 bar. Por ser uma molécula polar e, em condições ambientes, possuir a ligação de hidrogênio mais forte, seu estado supercrítico é marcado por características únicas e efeitos adicionais incomuns. Com o aumento da temperatura e pressão, a partir da temperatura ambiente, a densidade da água diminui causando dois efeitos principais: primeiramente, enfraquece de forma significativa as ligações de 


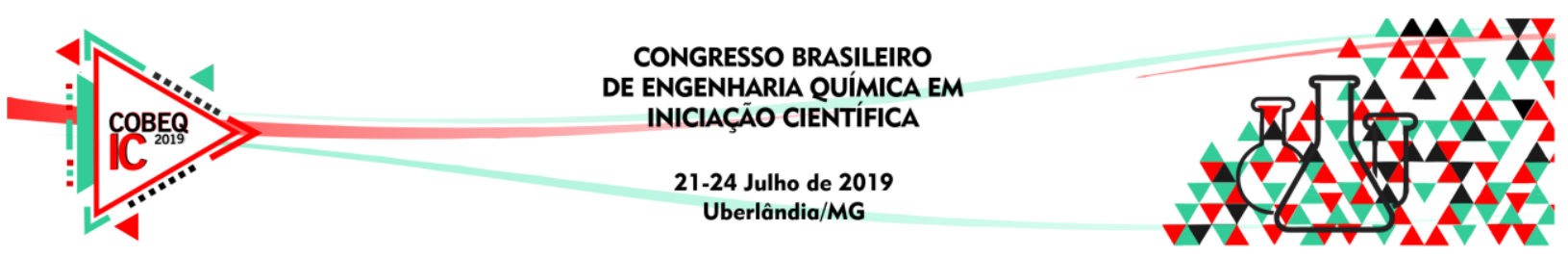

hidrogênio entre suas moléculas; em consequência, o afastamento das moléculas (volume vazio) diminui as interações dipolo-dipolo (KUTZ, 2007).

Nessas condições, a solubilidade dos eletrólitos inorgânicos diminui drasticamente, enquanto a solubilidade dos não-eletrólitos orgânicos aumenta exponencialmente (FRANCK, 1983). Dessa forma, compostos apolares como a maioria dos hidrocarbonetos e gases (Exemplos: $\mathrm{O}_{2}, \mathrm{~N}_{2}$ ) se tornam altamente solúveis. A constante de autoprotólise $(\mathrm{Kw})$ da água aumenta com a temperatura, logo, a água supercrítica (ASC) é ao mesmo tempo altamente ácida e básica. Sabe-se também, que a solubilidade de gases como hidrogênio e oxigênio aumentam, principalmente, por função da pressão.

Dessa forma, a ASC é capaz de reformar lixo orgânico, como o plástico, seja por degradação térmica ou oxidação dos compostos. Entretanto, altas temperaturas e o ambiente químico agressivo dessas reações levam a dois problemas encontrados em reações que utilizam ASC: precipitação/ acumulação de sal e corrosão (MARRONE \& HONG, 2009).

\section{DISCUSSÕES}

As mudanças que ocorrem na água ao atingir seu estado supercrítico podem ser expressas ao observar algumas propriedades físicas, como a constante dielétrica $(\varepsilon)$ e o produto iônico $\left(\mathrm{K}_{\mathrm{W}}\right)$. A constante dielétrica da água $(\varepsilon)$ tem um valor de 78.5 a temperatura ambiente $\left(25^{\circ} \mathrm{C}\right)$; Ao aumentar a temperatura o valor da constante diminui gradativamente e em $450^{\circ} \mathrm{C}$ a 250 bar, ele tem um valor próximo de 2 , o que é comparável ao de um solvente apolar tal como ciclohexano. O produto iônico da água $\left(\mathrm{K}_{\mathrm{W}}\right)$ começa na grandeza de $10^{-14}(\mathrm{~mol} / \mathrm{kg})^{2}$ nas condições ambientes e inicialmente aumenta até um máximo de cerca de $10^{-11}(\mathrm{~mol} / \mathrm{kg})^{2}$ no intervalo de 200 a $300^{\circ} \mathrm{C}$ antes de cair para $<10^{-23}(\mathrm{~mol} / \mathrm{kg})^{2}$ acima de $550^{\circ} \mathrm{C}(\mathrm{KUTZ}, 2007)$. Esta alteração de mais de 12 ordens de grandeza garante que a água seja, ao mesmo tempo, altamente ácida e básica, resultando um efeito significativo no papel das reações catalisadas por ácido / base, corrosão e capacidade de solubilizar sais (YAMANAKA, 2007).

À medida que a água é aquecida e pressurizada da condição subcrítica para supercrítica suas propriedades físicas sofrem mudanças nos valores, como viscosidade, difusibilidade e constante dielétrica. Dessa forma, a mais recente formulação de propriedades termodinâmicas da água, emitida pela Associação Internacional para as Propriedades da Água e Vapor (sigla em inglês: IAPWS-95), permite um cálculo razoavelmente preciso de um número de propriedades importantes em uma ampla gama de condições que abrangem do estado subcrítico à supercrítico (KUTZ, 2007).

O diagrama de fases da água é mostrado na Figura 2. A água supercrítica pode ser classificada em três categorias, regiões de baixa, média e alta densidade, devido à importância do parâmetro em relação às propriedades termodinâmicas e dinâmicas. Na região supercrítica, muitas propriedades físico-químicas da água podem ser representadas pelo único parâmetro, densidade, em vez dos parâmetros, temperatura e pressão (YAMANAKA; OHTAKI, 2007).

Na região de baixa densidade, a densidade da água, $\rho$, é menor que sua densidade crítica $\left(\rho<\rho_{c}\right)$. Em contrapartida, a densidade na região de densidade média é um intermediário entre 


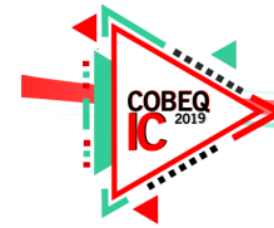

$\rho_{\mathrm{c}}$ e $1 \mathrm{~g} \cdot \mathrm{cm}^{-3}\left(\rho_{\mathrm{c}} \leq \rho \leq 1 \mathrm{~g} \cdot \mathrm{cm}^{-3}\right)$, enquanto que para a região de alta densidade, é maior do que $1 \mathrm{~g} . \mathrm{cm}^{-3}\left(1 \mathrm{~g} . \mathrm{cm}^{-3}<\rho\right)$ (YAMANAKA \& OHTAKI, 2007).

Figura 2 - Diagrama de fases da água.

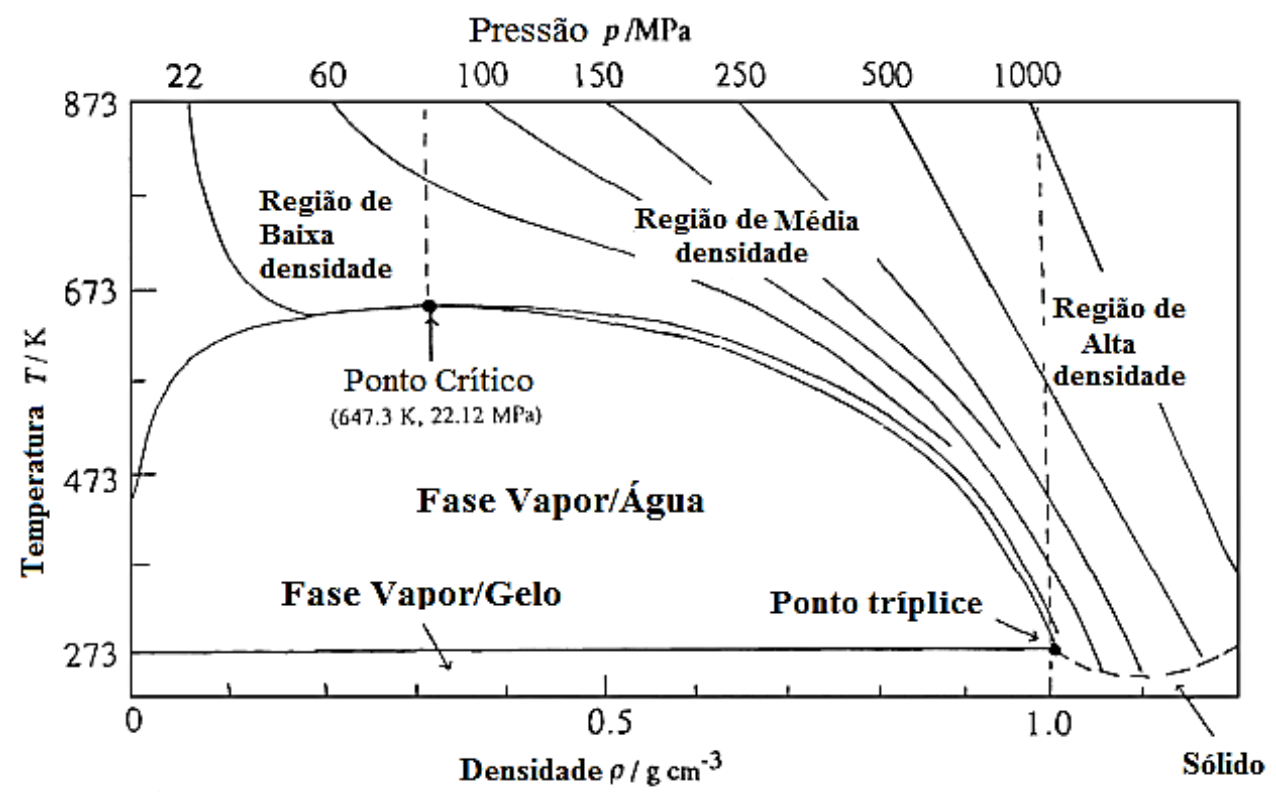

Fonte: Thermodynamics, Solubility and Environmental Issues.

A densidade do fluido no reator pode ser estimada utilizando softwares de simulação de processos para analisar equações de estado em dadas temperatura e pressão como demonstrado na Tabela 1. As equações de estado mais utilizadas com água supercrítica são: Redlich-KwongSoave (RK-SOAVE) e Schwartzentruber-Renon (SR-POLAR) (KUTZ, 2007).

Tabela 1 - Comparativo entre dados de propriedades termodinâmicas da água.

\begin{tabular}{cccccc}
\hline Modelo & $\mathrm{T}\left({ }^{\circ} \mathrm{C}\right)$ & $\mathrm{P}(\mathrm{bar})$ & $\begin{array}{c}v(\mathrm{cc} / \mathrm{mol}) \\
\text { Vapor }\end{array}$ & $\begin{array}{c}v(\mathrm{cc} / \mathrm{mol}) \\
\text { Líquido }\end{array}$ & $\begin{array}{c}\text { Densidade } \\
(\mathrm{g} / \mathrm{cc})\end{array}$ \\
\hline IAPWS-95 & 374 & 221 & 46,724 & 46,724 & 0,3856 \\
& 600 & 235 & 273,345 & 273,345 & 0,0659 \\
RK-SOAVE & 374 & 221 & 83,6618 & 55,9816 & 0,3218 \\
& 600 & 235 & 278,42 & 55,9816 & 0,0647 \\
SR-POLAR & 374 & 221 & 74,2669 & 74,2669 & 0,2426 \\
& 600 & 235 & 282,414 & 105,056 & 0,06379 \\
\hline
\end{tabular}

Fonte: Elaborado pelos próprios autores, 2018. 


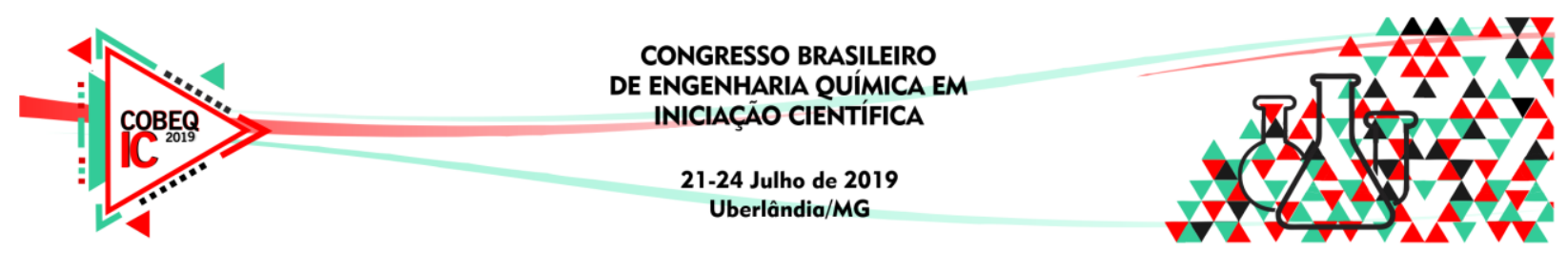

\subsection{O processo de oxidação com água supercrítica (OASC)}

No processo OASC, compostos orgânicos são reunidos com algum oxidante (geralmente oxigênio ou ar) e reagem em um meio rico em água acima de suas temperatura e pressão crítica. A alimentação orgânica pode ser pura ou em solução, sendo mais recomendado soluções aquosas com os produtos orgânicos diluídos. No caso de partículas sólidas, geralmente, faz-se necessário alguma forma de pré-tratamento, como a redução de tamanho (KUTZ, 2007).

Nas temperaturas e circunstâncias características da água supercrítica, compostos orgânicos são rapidamente oxidados em dióxido de carbono e água. Heteroátomos (Exemplos: $\mathrm{Cl}, \mathrm{P}, \mathrm{S}, \mathrm{N}$, etc.) presentes no material alimentado serão convertidos nos seus ácidos minerais correspondentes e gás nitrogênio (Exemplos: $\mathrm{HCl}, \mathrm{H}_{2} \mathrm{SO}_{4}, \mathrm{H}_{3} \mathrm{PO}_{4}, \mathrm{HNO}_{3}, \mathrm{~N}_{2}$, etc.). Os ácidos formados, geralmente, são neutralizados por uma solução básica injetada no reator levando à precipitação de sais. Metais incluídos nos resíduos são frequentemente oxidados em óxidos metálicos, que também são precipitados no reator. Estes, representam uma das dificuldades principais de se utilizar o processo OASC pois os sais, ácidos e óxidos metálicos são responsáveis pela corrosão e abrasão que ocorrem nas paredes internas do reator e o acúmulo de sais (FRANCK,1983).

Em condições típicas de operação do processo $\left(550-650^{\circ} \mathrm{C}\right.$ e $235-250$ bar) a decomposição do material orgânico pode ser maior que $99.00 \%$ e o tempo de residência varia de segundos a um minuto (YAMANAKA \& OHTAKI, 2007). Apesar do pouco tempo de residência pouco ou nenhum monóxido de carbono é formado, evidenciando uma oxidação completa e eficiência de processo de aproximadamente $100 \%$.

\section{CONCLUSÃO}

Diante da problemática acentuada na gestão de resíduos sólidos, sobretudo em relação ao crescimento exponencial do uso de materiais plásticos pela humanidade, se faz evidente a necessidade de estudos que tratem da diminuição desse uso e da sua reciclagem. O referente artigo traz um método limpo de degradação de cadeias poliméricas por meio da quebra do composto em conjunto da água em condições supercríticas. A partir dos estudos deste trabalho pode-se concluir que o método de degradação de compostos orgânicos via oxidação por água supercrítica pode ser uma alternativa promissora pois tem como subproduto um conjunto de moléculas menores de valor agregado, logo, mais estudos nesta área devem ser elaborados. 


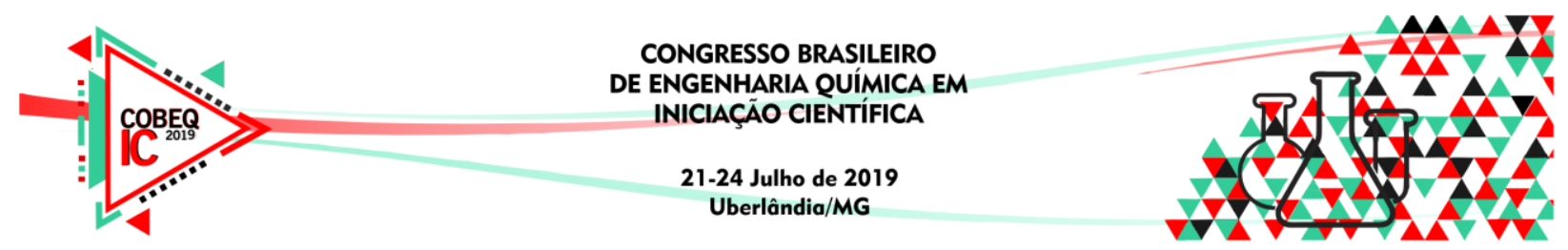

\section{REFERÊNCIAS}

FRANCK, E.u.. Thermophysical properties of supercritical fluids with special consideration of aqueous systems. Fluid Phase Equilibria, [s.1.], v. 10, n. 2-3, p.211-222, jan. 1983. Elsevier BV. http://dx.doi.org/10.1016/0378-3812(83)80035-1.

KORETSKY, Milo D.. Termodinâmica Para Engenharia Química. Rio de Janeiro: Livros Técnicos e Científicos Editora S.a., 2007. 520 p. Tradução de: Engineering and chemical thermodynamics;

KUTZ, Myer. Environmentally Conscious Materials and Chemicals Processing. Canada: John Wiley \& Sons, 2007. 500 p;

MARRONE, Philip A.; HONG, Glenn T.. Corrosion control methods in supercritical water oxidation and gasification processes. The Journal Of Supercritical Fluids, [s.1.], v. 51, n. 2, p.83-103, dez. 2009. Elsevier BV. http://dx.doi.org/10.1016/j.supflu.2009.08.001;

PANT, Deepak et al. Chemical and biological extraction of metals present in E waste: A hybrid technology. Waste Management, [s.1.], v. 32, n. 5, p.979-990, maio 2012. Elsevier BV. http://dx.doi.org/10.1016/j.wasman.2011.12.002.

YOUNG \& FREEDMAN. Física II: Termodinâmica e Ondas. $12^{\circ}$ ed.; São Paulo; Pearson, 2008.

YAMANAKA, Koji; OHTAKI, Hitoshi. Supercritical Fluids and Reductions in Environmental Pollution. In: LETCHER, Trevor M. (Ed.). Thermodynamics, Solubility and Environmental Issues. [s.I.]: Elsevier Science \& Technology Books, 2007. Cap. 4. p. 50-84; 